\title{
Flow behavior of UCM viscoelastic fluid in sudden contraction channel
}

\author{
Chunquan $\mathrm{Fu}^{1}$, Hongliang Zhou ${ }^{2}$, Hongjun Yin ${ }^{1 *}$, Huiying Zhong ${ }^{1}$, Haimei Jiang ${ }^{3}$ \\ ${ }^{1}$ Key Laboratory of Enhanced Oil and Gas Recovery Ministry of Educaion, Northeast Petroleum University, Daqing, China; \\ *Corresponding Author: yinhj7176@126.com \\ ${ }^{2}$ Daqing Oilfield Company Ltd., Daqing, China; 13836758851@163.com \\ ${ }^{3}$ China National Offshore Oil Company Research Center, Beijing, China; 3629461@163.com
}

Received 20 April 2010; revised 22 May 2010; accepted 26 May 2010.

\begin{abstract}
A finite volume method for the numerical solution of viscoelastic flows is given. The flow of a differential upper-convected Maxwell (UCM) fluid through a contraction channel has been chosen as a prototype example. The conservation and constitutive equations are solved using the finite volume method (FVM) in a staggered grid with an upwind scheme for the viscoelastic stresses and a hybrid scheme for the velocities. An enhanced-in-speed pressure-correction algorithm is used and a method for handling the source term in the momentum equations is employed. Improved accuracy is achieved by a special discretization of the boundary conditions. Stable solutions are obtained for higher Weissenberg number (We), further extending the range of simulations with the FVM. Numerical results show the viscoelasticity of polymer solutions is the main factor influencing the sweep efficiency.
\end{abstract}

Keywords: Upper-Convected Maxwell (UCM) Model; Finite Volume Method; Viscoelasticity; Sweep Efficiency

\section{INTRODUCTION}

In the recent years, numerical simulation of viscoelastic flows has been a powerful tool for understanding the fluid behavior in a variety of processes of both industrial

Project supported by the 973 National Basic Research Program of China (Grant No: 2005cb221304), by Heilongjiang Provincial Science and Technology Plan Project (Grant No: GZ09A407) and by the Research Program of Innovation Team of Science and Technology in Enhanced Oil and Gas Recovery (Grant No: 2009td08) and scientific interest $[1,2]$. Polymeric fluids, owing to their viscoelastic characters, are of particular interest in the numerical simulation because of their wide applications in material processing and their different behavior from that of Newtonian fluids in ways which are often complex and striking. Although there have been many successful numerical predictions of elastic fluid flows [3-5], in which the Weissenberg number (We), standing for the elasticity, is low.

In the process of water flooding alone, the residual oil remaining within porous media is difficult to be displaced or recovered. In comparison, polymer flooding is more effective. Experimental results $[2,5,6]$ indicated the viscoelasticity of polymer solutions can enhance the displacement efficiency, but there have been few theoretical studies on this subject.

In this work, with the upper-convected Maxwell (UCM) model, the fluid flow through a 4:1 sudden contraction channel is studied by using a stable finite volume scheme. An enhanced-in-speed pressure correction algorithm and a method for handling the source term in the momentum equations are employed. The simulation accuracy is improved by a special discretization of the boundary conditions. The presented method succeeds in providing accurate numerical solutions, and elasticity levels up to $W e=3.0$. Where with the finite difference method the $W e$, standing for the elasticity, is less than 0.5 $[7,8]$.

In the following sections, the description of the problem, the mathematical model of the flow and the solution method are described respectively. The discretization of the source term and the boundary conditions are separately examined. The contours of velocity, stream function and pressure are drawn. Finally, the simulation results are presented and conclusions are drawn regarding the use of the FVM for viscoelastic flow simulations. 


\section{MATHETICL MODEL}

\subsection{The Model of Sudden Contraction Channel}

The micro-pores of an actual reservoir are in general complicated. These pores are often simplified in numerical simulation. The problem geometry is shown in Figure 1. It concerns the flow of a UCM fluid through a planar 4:1 sudden contraction channel. Then, flow behavior of viscoelastic polymer solutions is studied with this simplified physical model. Note that the dimension in the Figure 1 are dimensionless.

\subsection{Governing Equations}

The isothermal flow through contraction for incompressible fluids, such as polymer solutions and melts, is governed by the equation of continuity and motion, which can be expressed as

$$
\begin{gathered}
\nabla \cdot v=0 \\
\rho v \cdot \nabla v=-\nabla p+\nabla \cdot \tau
\end{gathered}
$$

where $v$ is the velocity vector, $p$ the pressure, $\tau$ the extra stress tensor and $\rho$ the density.

The constitutive equation that relates the stresses $\tau$ to the deformation history is predescribed by the UCM model, which in its differential form is written as

$$
\tau+\lambda \stackrel{\nabla}{\tau}=\mu \dot{\gamma}
$$

where $\lambda$ is the relaxation time, $\mu$ a constant viscosity, $\dot{\gamma}$ the rate-of-strain tensor and $\stackrel{\nabla}{\tau}$ Oldroyd's upper convected derivative of the stress tensor $\tau$.

The above equations are non dimensionalized by introducing the non-dimensional variables

$$
\begin{aligned}
& x_{\mathrm{D}}=\frac{x}{L}, y_{\mathrm{D}}=\frac{y}{L}, \tau_{\mathrm{D}}=\frac{L}{\eta U} \tau, \\
& u_{\mathrm{D}}=\frac{u}{U}, v_{\mathrm{D}}=\frac{v}{U}, p_{\mathrm{D}}=\frac{L}{\eta U} p .
\end{aligned}
$$

where the characteristic velocity $(U)$ and characteristic length $(L)$ are taken as the average velocity in the down-

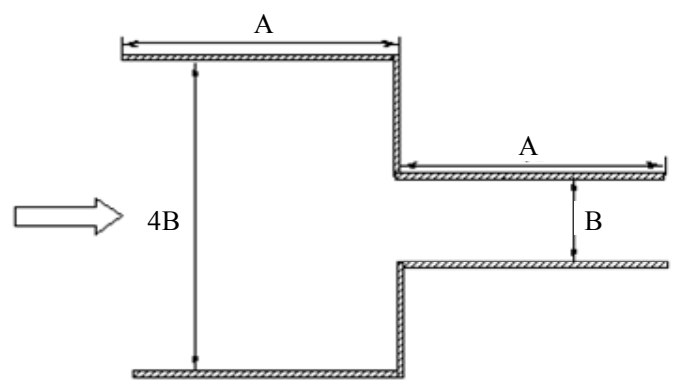

Figure 1. The model of sudden contraction channel. stream half channel and the width of the downstream half channel, respectively, $\eta$ is the constant shear viscosity, $u$ is the velocity component in the $x$ direction, and $v$ is the velocity component in the $y$ direction.

Therefore, in the dimensionless form the governing equations are given in the following, where the subscript $D$ is omitted for brevity.

For a two-dimensional system in a rectangular co-ordinates $(x, y)$ with the velocity components $(u, v)$, the continuity Eq.1 can be written as

$$
\frac{\partial u}{\partial x}+\frac{\partial v}{\partial y}=0
$$

The momentum equation (Eq.2) is given by

$$
\begin{aligned}
& \operatorname{Re}\left[\frac{\partial}{\partial x}(u u)+\frac{\partial}{\partial y}(u v)\right]=-\frac{\partial p}{\partial x}+\frac{\partial \tau_{x x}}{\partial x}+\frac{\partial \tau_{x y}}{\partial y} \\
& \operatorname{Re}\left[\frac{\partial}{\partial x}(v v)+\frac{\partial}{\partial y}(v u)\right]=-\frac{\partial p}{\partial y}+\frac{\partial \tau_{x y}}{\partial x}+\frac{\partial \tau_{y y}}{\partial y}
\end{aligned}
$$

and the constitutive equations for the UCM fluid can be written as

$$
\begin{gathered}
W e\left[\frac{\partial}{\partial x}\left(u \tau_{x x}\right)+\frac{\partial}{\partial y}\left(v \tau_{x x}\right)\right] \\
=2 \frac{\partial u}{\partial x}-\left(1-2 W e \frac{\partial u}{\partial x}\right) \tau_{x x}+2 W e \frac{\partial u}{\partial y} \tau_{x y} \\
W e\left[\frac{\partial}{\partial x}\left(u \tau_{y y}\right)+\frac{\partial}{\partial y}\left(v \tau_{y y}\right)\right] \\
=2 \frac{\partial v}{\partial y}-\left(1-2 W e \frac{\partial v}{\partial y}\right) \tau_{y y}+2 W e \frac{\partial v}{\partial x} \tau_{x y} \\
W e\left[\frac{\partial}{\partial x}\left(u \tau_{x y}\right)+\frac{\partial}{\partial y}\left(v \tau_{x y}\right)\right] \\
=\left(\frac{\partial v}{\partial x}+\frac{\partial u}{\partial y}\right)-\tau_{x y}+W e\left(\frac{\partial v}{\partial x} \tau_{x x}+\frac{\partial u}{\partial y} \tau_{y y}\right)
\end{gathered}
$$

where $\tau_{x x}, \tau_{x y}$ and $\tau_{y y}$ are the stress components in usual sense.

The Weissenberg number (We) and Reynolds number $(R e)$ in Eq.9 are defined by

$$
W e=\lambda \frac{U}{L}, R e=\frac{\rho U L}{\eta} .
$$

To solve Eqs.4-9, the boundary and initial conditions are given below.

For the full-developed steady Poiseuille flow, the inlet boundary condition is

$$
\begin{gathered}
u=6\left(\frac{1}{4}-y^{2}\right), v=0, \tau_{x x}=2 W \mathrm{e}\left(\frac{\partial u}{\partial y}\right)^{2}, \\
\tau_{y y}=0, \tau_{x y}=\frac{\partial u}{\partial y} .
\end{gathered}
$$

No-slip conditions are imposed on solid boundaries 
and symmetry conditions are specified on the symmetrical axis [9].

At the outlet a fully developed velocity profile is imposed with the homogeneous Neumann boundary conditions for the extra-stress, i.e.,

$$
\frac{\partial \tau_{x x}}{\partial x}=\frac{\partial \tau_{x y}}{\partial x}=\frac{\partial \tau_{y y}}{\partial x}=0
$$

\section{NUMERICAL ALGORITHM}

The constitutive relation Eq.3 is solved together with Eqs.1 and 2 using the FVM. Here some details about our own implementation of the method are given.

\subsection{Computational Grid}

A grid is placed in the computational domain and a control volume is associated with each unknown on the grid. This grid, called the reference grid, remains fixed in space for all time. In this study, we assume that the sides of each control volume are aligned with the coordinate axes. Each component is integrated over an appropriate control volume [10]. The grid is shown in Figure 2.

The staggered grid is used in which the different dependent variables are approximated at different mesh points. Both meshes ensure that the solution is not polluted by spurious pressure modes. On a non-staggered mesh the familiar chequerboard mode is applied.

\subsection{Discretization}

A simple finite volume formulation is used for the discretization and the first-order Euler implicit formula is used for temporal differences because of its simplicity for implementation and unconditional stability in numerical computations.

In employing the FVM, the governing equations are written in the following general form [11]:

$\nabla \cdot(m v \varphi)=\nabla \cdot(\Gamma \nabla \varphi)+S$

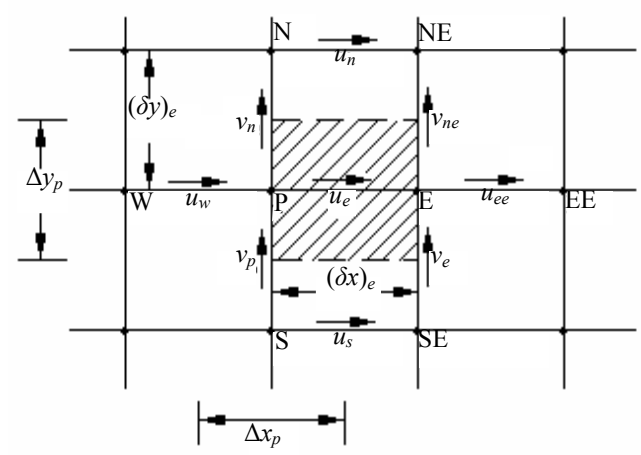

Figure 2. Staggered grid.

\subsubsection{Discretization of Continuity Equations}

The discretized continuity equation reflects the mass conservation for each cell:

$$
F_{\mathrm{e}}-F_{\mathrm{w}}+F_{\mathrm{n}}-F_{\mathrm{s}}=0
$$

where

$$
\begin{gathered}
F_{\mathrm{e}}=\operatorname{Reu}_{\mathrm{e}} \Delta y, F_{\mathrm{w}}=\operatorname{Reu}_{\mathrm{w}} \Delta y, \\
F_{\mathrm{n}}=\operatorname{Reu}_{\mathrm{n}} \Delta x, \quad F_{\mathrm{s}}=\operatorname{Reu}_{\mathrm{s}} \Delta x .
\end{gathered}
$$

$F_{\mathrm{e}}$ is the outgoing mass flow rates at cell face e, $u_{\mathrm{e}}$ refers to the cell face velocity component, and the same for $F_{\mathrm{w}}$, $F_{\mathrm{n}}, F_{\mathrm{s}}$ and $u_{\mathrm{w}}, u_{\mathrm{n}}, u_{\mathrm{s}}$.

\subsubsection{Discretization of Momentum Equations}

Eqs.5 and 6 can be written in the general form of Eq.10 using the transformation

$$
\begin{aligned}
& \tau_{x x}^{\prime}=\tau_{x x}-2 \eta \frac{\partial u}{\partial x} \\
& \tau_{y y}^{\prime}=\tau_{y y}-2 \eta \frac{\partial v}{\partial y} \\
& \tau_{x y}^{\prime}=\tau_{x y}-\eta\left(\frac{\partial u}{\partial y}+\frac{\partial v}{\partial x}\right)
\end{aligned}
$$

where $\tau^{\prime}$ is the elastic part of the stress tensor $\tau$ [12].

Substituting Eqs.11-13 into the momentum equations and assuming a constant viscosity turn Eqs.5 and 6 into

$$
\begin{gathered}
\frac{\partial}{\partial x}(\operatorname{Re} u u)+\frac{\partial}{\partial y}(\operatorname{Re} u v)=\frac{\partial}{\partial x}\left(\frac{\partial u}{\partial x}\right)+\frac{\partial}{\partial y}\left(\frac{\partial u}{\partial y}\right)+ \\
\frac{\partial \tau_{x x}^{\prime}}{\partial x}+\frac{\partial \tau_{x y}^{\prime}}{\partial y}+\frac{\partial}{\partial x}\left(\frac{\partial u}{\partial x}+\frac{\partial v}{\partial y}\right)-\frac{\partial p}{\partial x} \\
\frac{\partial}{\partial x}(\operatorname{Re} v v)+\frac{\partial}{\partial y}(\operatorname{Re} v u)=\frac{\partial}{\partial x}\left(\frac{\partial v}{\partial x}\right)+\frac{\partial}{\partial y}\left(\frac{\partial v}{\partial y}\right)+ \\
\frac{\partial \tau_{y y}^{\prime}}{\partial y}+\frac{\partial \tau_{x y}^{\prime}}{\partial x}+\frac{\partial}{\partial x}\left(\frac{\partial u}{\partial x}+\frac{\partial v}{\partial y}\right)-\frac{\partial p}{\partial y}
\end{gathered}
$$

In Eqs.15 and 16 the viscous parts are discretized as the diffusion terms of Eq.10, while the other terms on the right-hand side are treated as extra source terms.

Then the final discretized equations of momentum can be expressed symbolically in a general form:

$$
a_{\mathrm{P}} u_{\mathrm{P}}=a_{\mathrm{E}} u_{\mathrm{E}}+a_{\mathrm{W}} u_{\mathrm{W}}+a_{\mathrm{N}} u_{\mathrm{N}}+a_{\mathrm{S}} u_{\mathrm{S}}+S_{\mathrm{u}}
$$

where $u_{\mathrm{P}}$ refers to the cell velocity component, and the same for $u_{\mathrm{E}}, u_{\mathrm{W}}, u_{\mathrm{N}}$ and $u_{\mathrm{S}}$.

$$
\begin{aligned}
& a_{\mathrm{E}}=\frac{\Delta y}{\delta x_{\mathrm{e}}}+\max \left(-F_{\mathrm{e}}, 0\right), \\
& a_{\mathrm{W}}=\frac{\Delta y}{\delta x_{\mathrm{w}}}+\max \left(F_{\mathrm{w}}, 0\right),
\end{aligned}
$$




$$
\begin{gathered}
a_{\mathrm{N}}=\frac{\Delta x}{\delta y_{\mathrm{n}}}+\max \left(-F_{\mathrm{n}}, 0\right) \\
a_{\mathrm{S}}=\frac{\Delta x}{\delta y_{\mathrm{s}}}+\max \left(F_{\mathrm{s}}, 0\right) .
\end{gathered}
$$

\subsubsection{Discretization of Constitutive Equations}

The adopted viscoelastic model also has the general transport equation from Eq.10 without diffusion term $(\Gamma=0)$. To ensure numerical stability, generally, a firstorder upwind difference (UD) is used for spatial discretization. Thus, the discretized constitutive equation can be written as

$$
a_{i \mathrm{P}}^{\tau} \tau_{i j \mathrm{P}}=a_{\mathrm{E}}^{\tau} \tau_{i j \mathrm{E}}+a_{\mathrm{W}}^{\tau} \tau_{i j \mathrm{~W}}+a_{\mathrm{N}}^{\tau} \tau_{i j \mathrm{~N}}+a_{\mathrm{S}}^{\tau} \tau_{i j \mathrm{~S}}+S_{\tau i j}
$$

where $\tau_{i j \mathrm{P}}$ refers to the cell stress component, and the same for $\tau_{i j \mathrm{E}}, \tau_{i j \mathrm{~W}}, \tau_{i j \mathrm{~N}}$ and $\tau_{i j \mathrm{~S}}$.

$$
\begin{aligned}
& a_{\mathrm{E}}^{\tau}=W e \max \left(-F_{\mathrm{e}}, 0\right), \quad a_{\mathrm{W}}^{\tau}=W e \max \left(F_{\mathrm{w}}, 0\right), \\
& a_{\mathrm{N}}^{\tau}=W e \max \left(-F_{\mathrm{n}}, 0\right), \quad a_{\mathrm{S}}^{\tau}=W e \max \left(F_{\mathrm{s}}, 0\right), \\
& a_{\mathrm{P}}^{\tau}=\Delta x \Delta y+a_{\mathrm{E}}^{\tau}+a_{\mathrm{W}}^{\tau}+a_{\mathrm{N}}^{\tau}+a_{\mathrm{S}}^{\tau} \\
& S_{\tau_{X X}}=\left(\frac{4}{3}+2 W e \tau_{x x}\right)\left(u_{\mathrm{e}}-u_{\mathrm{w}}\right) \Delta y+ \\
& 2 W e \tau_{x y}\left(u_{\mathrm{n}}-u_{\mathrm{s}}\right) \Delta x-\frac{2}{3}\left(v_{\mathrm{n}}-v_{\mathrm{s}}\right) \\
& S_{\tau_{x y}}=\left(1+W e \tau_{y y}\right)\left(u_{\mathrm{n}}-u_{\mathrm{s}}\right)+W e \tau_{x y}\left(v_{\mathrm{n}}-v_{\mathrm{s}}\right)+ \\
& W e \tau_{x y}\left(u_{\mathrm{e}}-u_{\mathrm{w}}\right)+\left(1+W e \tau_{x x}\right)\left(v_{\mathrm{e}}-v_{\mathrm{w}}\right) \\
& S_{\tau_{y y}}=-\frac{2}{3}\left(u_{\mathrm{e}}-u_{\mathrm{w}}\right)+2 W e \tau_{x y}\left(v_{\mathrm{e}}-v_{\mathrm{w}}\right)+ \\
& \left(\frac{4}{3}+2 W e \tau_{y y}\right)\left(v_{\mathrm{n}}-v_{\mathrm{s}}\right)
\end{aligned}
$$

where $v_{\mathrm{e}}$ refers to the cell face velocity component, and the same for $v_{\mathrm{w}}, v_{\mathrm{n}}, v_{\mathrm{s}}$.

\subsection{Solution of the Discretized Equations}

In non-linear problems the equations are solved with iterative methods using an initial guess for the primitive variables and giving an approximate solution. The iterative methods have the advantage of less computer memory as they take advantage of zero elements in the coefficient matrix. In this work, the strongly implicit procedure (SIP) [13-15] is used, which involves the direct, simultaneous solution of the set of equations formed by modification of the original matrix equation. The modified matrix is constructed according to two criteria: 1) the equation set must remain more strongly implicit than in the alternating direction implicit (ADI) case; and 2) the elimination procedure for the modified set must be efficient.

\section{RESULTS AND DISCUSSION}

As discussed above, a numerical simulation method is used and the stream function contour, velocity contour and pressure contour with different $W e$ can be obtained. As an example, the stream function and velocity contours with $W e$ equates 0 to 3.0 are shown in Figures 3-5, respectively.

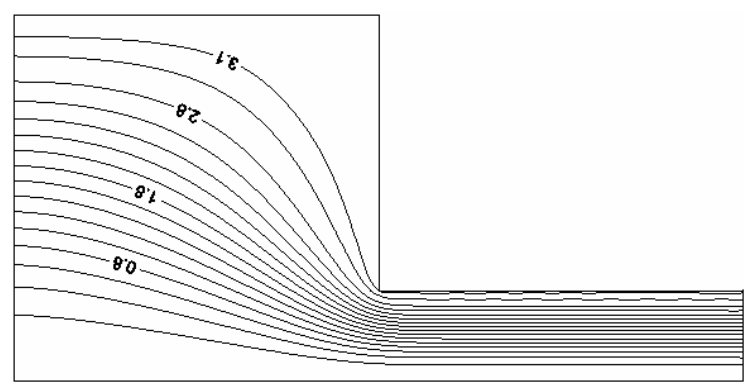

(a) $W e=0, R e=10^{-5}$

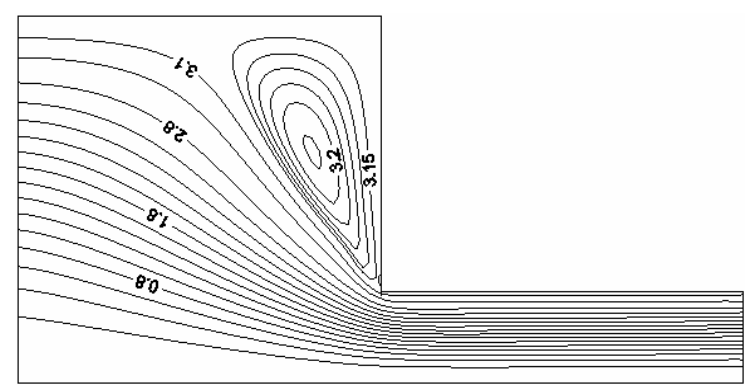

(b) $\mathrm{We}=0.6, \operatorname{Re}=10^{-5}$

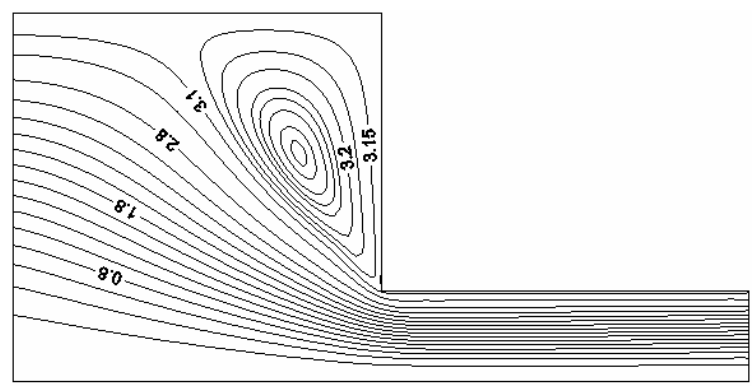

(c) $W e=1.5, R e=10^{-5}$

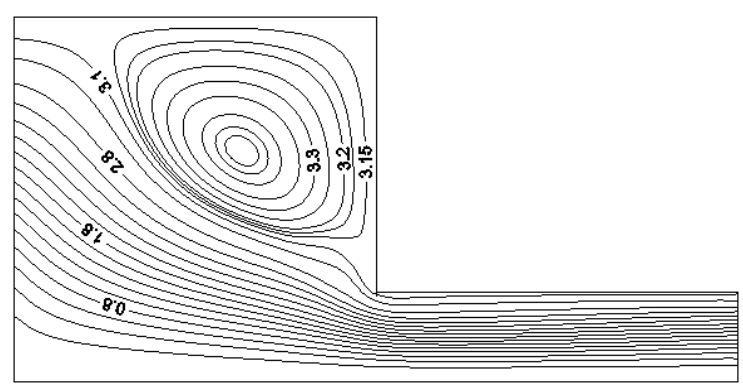

(d) $\mathrm{We}=3.0, \mathrm{Re}=10^{-5}$

Figure 3. Stream function contours. 
Figure 3 shows the influence of the $W e$ on the stream function, we can see that when the Reynolds number is smaller, the vortex area is expanding as $W$ e increasing, and the corresponding vortex strength will be enhanced, thereby the fluid velocity and applied force in the convex corner will increase too. So, displacing the dead oil in convex corner, enhance vortex-convex is an important reason to raise the angle of displacement oil. This is be-

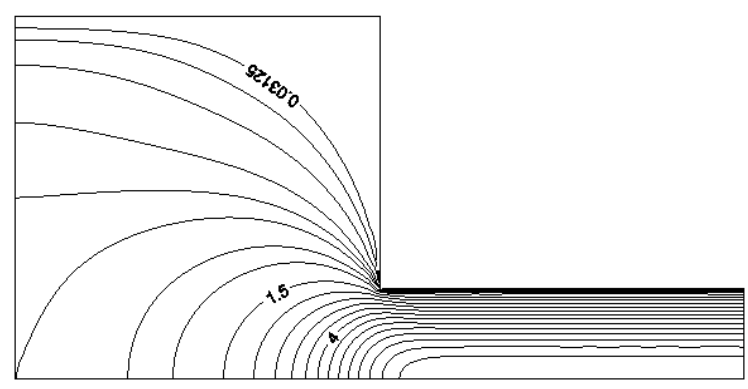

(a) $W e=0, R e=10^{-5}$

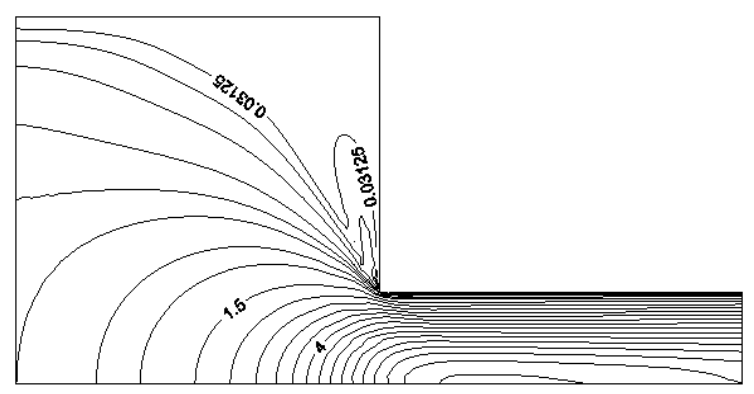

(b) $W e=0.6, R e=10^{-5}$

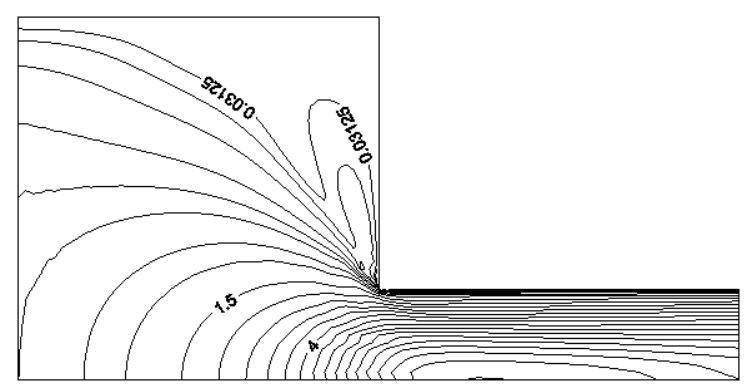

(c) $W e=1.5, R e=10^{-5}$

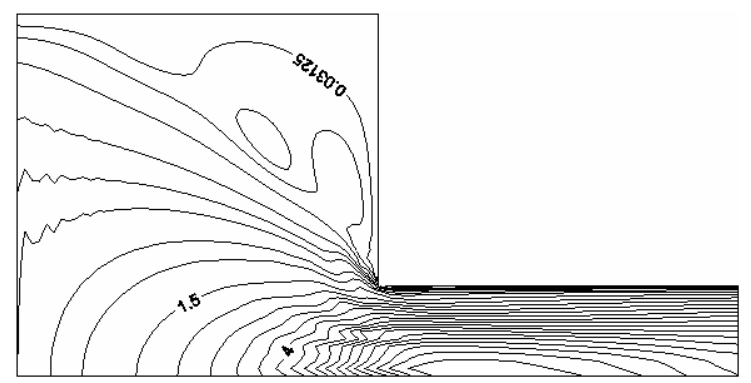

(d) $\mathrm{We}=3.0, \mathrm{Re}=10^{-5}$

Figure 4. Velocity contours. cause under the flowing conditions of reservoir (That is, Reynolds number is smaller), the viscoelastic of fluid plays a important role in fluid flow, the stronger the viscoelastic (That is, We is larger), the stronger the viscoelastic vortex is.

In Figure 4, from the area surrounded by the speed of $v=0.03125$, it is seen that the micro sweep area and sweep efficiency increase as the $W e$ increases.

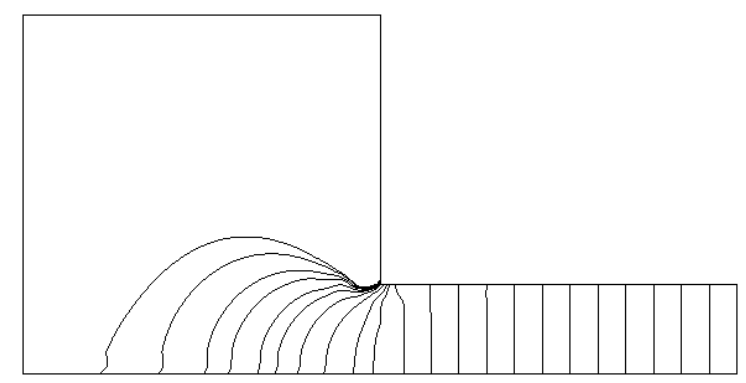

(a) $\mathrm{We}=0, \operatorname{Re}=10^{-5}$

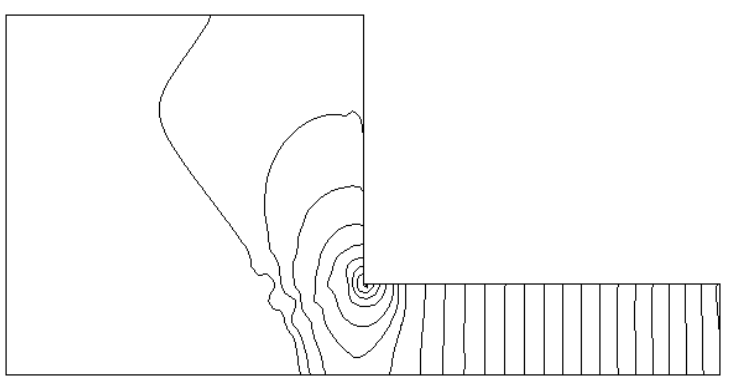

(b) $W e=0.6, R e=10^{-5}$

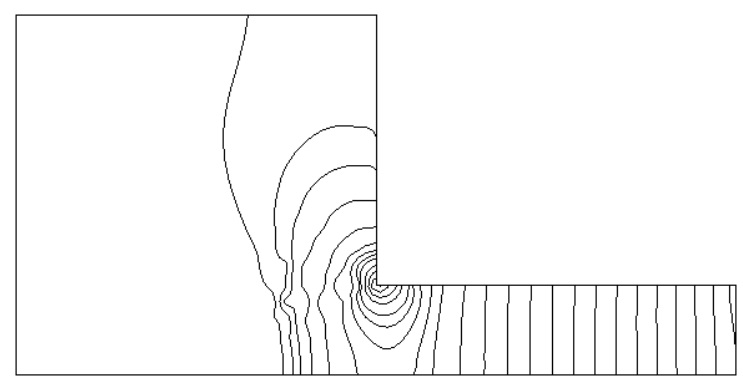

(c) $\mathrm{We}=1.5, \mathrm{Re}=10^{-5}$

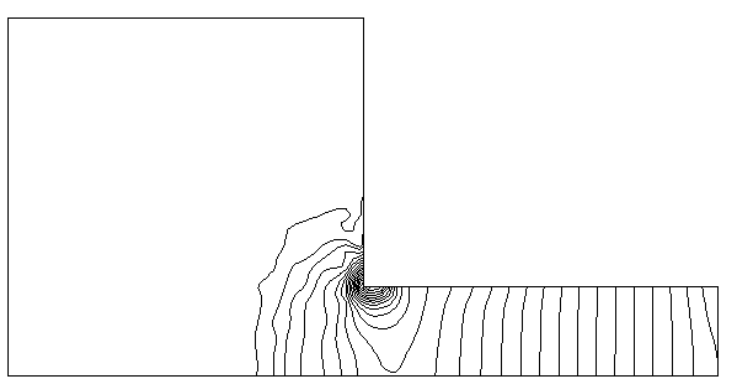

(d) $\mathrm{We}=3.0, \mathrm{Re}=10^{-5}$

Figure 5. Pressure contours. 
In Figure 5, at the downstream of the contraction, the pressures change gently. In the convex area of contraction, pressures vary intensely and the difference grows larger with the increase of We. That is to say, the pressure loss mainly happens at corner. The the pressure drop is larger with a bigger We. The high pressure drop and high velocity of viscoelastic polymer solution at corner strengthen the displacement and wash action, and it will increase the microscopic sweep efficiency.

\section{CONCLUSIONS}

In this paper, the flow of a UCM model fluid through a 4:1 sudden contraction channel has been studied using a stable finite volume scheme. The solution method succeeds in obtaining accurate values for all variables at elasticity levels up to $W e=3.0$.

The present simulations reinforce the point that the FVM can be used as a viable alternative for the solution of viscoelastic problems. The results are accurate and offer an improvement over previous numerical solutions. Although the present study has been applied to a UCM fluid in a relatively simple geometry, it can be further extended to other more realistic constitutive equations, such as the Phan-Thien-Tanner or Giesekus-Leonov models, etc. and to other geometries encountered in polymer processing.

Numerical results show that the viscoelasticity of polymer solutions is the main factor influencing sweep efficiency. With increasing elasticity, the flowing area in the corner is enlarged significantly, thus the area with immobile zones becomes smaller. Flow velocity is larger than that for a Newtonian fluid, the sweep area and displacement efficiency increase as the elasticity increases. The pressure drop in the convex area is larger with a bigger elasticity, and it will strengthen the displacement and wash action at the corner. The viscoelastic behavior of the displacing polymer fluids can in general improve the displacement efficiency in pores compared to using Newtonian fluids. This conclusion should be useful in selecting polymer fluids and designing polymer flooding operations.

\section{REFERENCES}

[1] Zhang, L.J. and Yue, X.A. (2007) Mechanism for visco- elastic polymer solution percolating through porous media. Journal of Hydrodynamics Series B, 19(2), 241248.

[2] Wang, D.M. and Lin, J.Z. (2008) Influence of the microforce produced by viscoelastic displacement of liquid on displacement efficiency. Journal of Xi'an Shiyou University (Natural Science Edition), 23(1), 4355.

[3] Yin, H.J., Wang, D.M. and Zhong, H.Y. (2006) Study on flow behaviors of viscoelastic. polymer solution in micropore with dead end. SPE 101950, San Antonio, Texas, 786-795.

[4] Yin, H.J. and Zhong, H.Y. (2007) Numerical simulations of viscoelastic flows through one slot Channel. Jour- nal of Hydrodynamics, Series B, 19(2), 210-216.

[5] Wang, D.M., Cheng, J.C. and Yang, Q.Y. (2000) Viscouselastic polymer can increase in cores. SPE 63227, Dallas, Texas, 719-728.

[6] Huang, Y.Z., Yu, D.S. and Zhang, G.F. (1990) The study on micro polymer flooding mechanism. Oilfield Chemistry, 3, 57-60.

[7] Yin, H.J. and Jiang, H.M. (2008) Bhavior of SPTT viscoelastic fluid in contraction channel. Petroleum Geology \& Oilfield Development in Daqing, 27(2), 5659.

[8] Zhang, L.J. (2001) Flow of voscoelastic fluid throught complex pores and its effect on microscopic displacement efficiency. Daqing Peturelum Institute, 37-37.

[9] Aboubacar, M., Matallah, H. and Webster, M.F. (2002) Highly elastic solutions for Oldroyd-B and Phan-Thien/ Tanner fluids with a finite volume/element method: Planar contraction flows. Non-Newtonian Fluid Mech, 103(1), 65-103.

[10] Jiang, H.M. (2009) The study on microscopic porous flow behavior of polymer solutions. Daqing Peturelum Institute, 10-26.

[11] Patankar, S.V. (1980) Numerical heat transfer and fluid flow [M]. New York, Hemisphere.

[12] Deng, J., Ren, A.L. and Zou, J.F. (2006) Threedimensional flow around two tandem circular cylinders with various spacing at $\mathrm{Re}=200$. Journal of Hydrodynamics, Series $B, \mathbf{1 8}(\mathbf{1}), 48-54$.

[13] Bao, F.B., Lin, J.Z. and Liu, Y.H. (2006) Research on the flow property in three dimensional cavity of microchannel. Journal of Hydrodynamics, Series B, 18(1), 20-25.

[14] Qu, J.P. and Xia, G.F. (1998) Reaserch on elastic behaviors of LDPE melt during capillary dynamic extrusion. Journal of South China University of Technology (Natural Science), 11, 76-80.

[15] Fortin, A. and Zine, A. (1992) An improved GARES method for solving viscoelastic fluid flow problems. Non-Newtonian Fluid Mechanics, 42(1-2), 1-18. 